Sādhanā Vol. 29, Part 6, December 2004, pp. 589-604. (C Printed in India

\title{
Techniques based on genetic algorithms for large deflection analysis of beams
}

\author{
RAJESH KUMAR $^{1}$, L S RAMACHANDRA ${ }^{1}$ and D ROY ${ }^{2 *}$ \\ ${ }^{1}$ Department of Civil Engineering, Indian Institute of Technology, \\ Kharagpur 721 302, India \\ ${ }^{2}$ Department of Civil Engineering, Indian Institute of Science, \\ Bangalore 560 012, India \\ e-mail: royd@civil.iisc.ernet.in
}

MS received 28 May 2004; revised 10 June 2004

\begin{abstract}
A couple of non-convex search strategies, based on the genetic algorithm, are suggested and numerically explored in the context of large-deflection analysis of planar, elastic beams. The first of these strategies is based on the stationarity of the energy functional in the equilibrium state and may therefore be considered weak. The second approach, on the other hand, attempts to directly solve the governing differential equation within an optimisation framework and such a solution may be thought of as strong. Several numerical illustrations and verifications with 'exact' solutions, if available, are provided.
\end{abstract}

Keywords. Genetic algorithms; large deflection analysis; analysis of beams.

\section{Introduction}

The beam is one of the most common structural elements used in a variety of aerospace, civil and mechanical engineering structures. Linear beam theories based on a linear momentcurvature relationship cannot be used for beams undergoing large deflections i.e., in which squares of the slopes are comparable to unity. Linear and linearization theories (based on tangent space approximations) at best are first-order approximations. As Stoker (1992) pointed out, "linearizations commonly made use of as reasonable or satisfactory approximations are simply a confession of defeat in the face of challenges presented by nonlinear systems". In dealing with the nonlinear behaviour of deformable bodies such as beams, the relationship between the extensional strains and the displacement components are taken to be nonlinear, resulting in nonlinear strain-displacement relations. This is the type of nonlinearity most commonly found in structures. The post-buckling analysis of cantilever columns subjected to edge concentrated load/axial load has been investigated by Barten (1945) and others (Bishop $\&$ Drucker 1945; Kerr 1964). Post-buckling analysis of uniform beams/columns under concentrated load at the free end is discussed by Timoshenko \& Gere (1961) in which the solution

\footnotetext{
${ }^{*}$ For communication
} 
is obtained in terms of elliptical integrals. Later on Mattiasson (1981) has reported the large deflection response of beam and frame problems in terms of first and second kinds of elliptic integrals. He has also described a technique for evaluating the numerical integrals. Lee et al (1993) have obtained the deformed configuration of the variable cross section cantilever beam subjected to the combined action of tip concentrated load, tip moment and linearly distributed lateral loads by numerical methods. Their beam model is based on Bernoulli-Euler beam theory. These authors have used Runge-Kutta and Regula-Falsi methods to solve the governing nonlinear differential equations. Kuznetsov \& Levyakov (2002) have obtained the post-critical equilibrium forms of simply supported columns subjected to axial load numerically. For this well-known problem they have shown the existence of bifurcation points and branches of the nonlinear solution. Recently, Vaz \& Silva (2003) have provided a formulation and solution procedure of slender rods subjected to axial terminal forces. They have shown that post-critical solutions are highly dependent on the boundary conditions of the bar. Nonlinear governing differential equations are solved by a two-point shooting technique. Ramachandra \& Roy (2001a, 2001b) have also adopted shooting strategies to solve a class of nonlinear boundary value problems in solid mechanics and have employed the transversal linearization method of integration. Some research effort has also been made to apply the kinematically exact 3-D beam theory (Simo \& Vu Quoc 1986; Simo 1992) in the context of post-buckling analyses of beams (Ibrahimbegovic 1996).

Equilibrium configurations of nonlinear beams under axial or transverse loads may be obtained by any of the nonlinear optimization methods available to solve the engineering optimization problems. Performance of these optimization strategies is best tested, in the context of problems in structural mechanics, against certain classical solutions, which are available in closed form for a class of nonlinear beam problems (Mattiason 1981). Among the range of available non-gradient methods (Rao 1984; Arora 1989), the genetic algorithm is one of the most popular non-convex global search techniques. In this study, two different classes of numerical techniques are developed and explored for large deflection problems in structural mechanics. The first approach is based on a minimization of the associated total potential energy whereas the second approach converts the governing boundary value problem (BVP) into a constrained initial value problem (IVP) using a shooting principle. The methodologies of the first and second approaches are presented in $\S 2$. In $\S 3$, numerical results obtained via the present approaches and comparisons with some of the classical results are provided. A summary of observations is presented in $\S 4$.

\section{The methodology}

This paper explores two different approaches, based on genetic algorithms, to search for equilibrium configurations of beams with different boundary conditions. While the first approach is based on a minimization of the total potential energy of the system, the second approach is via a constrained initial value formulation and direct conditional integration of the associated nonlinear differential equations. Use of either of the methods generally leads to a constrained optimization problem, which in turn is solved using the genetic search algorithm. In the first method a convergent series solution is assumed to represent the deflected shape of the beam. The undetermined amplitudes constitute the generalized co-ordinates associated with the asymptotic series solution. The generalized amplitudes, which minimize the potential energy of the elastic system, are then sought. Here it may be noted that global displacement function, which satisfies equilibrium of the structure, corresponds to the minimum value of the total potential. 
In the second method, a two-point boundary value problem is converted into a constrained initial value problem by treating the unknown initial conditions as variables to be so determined that the known boundary conditions at other boundary point are optimally satisfied. In other words, a direct integration of the governing differential equations has to be done, starting at one of the boundaries, in such a way that the errors on known conditions at the other boundary reduce to a minimum. The second method turns out to be computationally severalfold faster without any loss of accuracy. In the present study, numerical illustrations for the above procedures include determination of large static deflections and nonlinear post buckling equilibrium paths of beams (elastica problems, Timoshenko \& Gere 1963) with different boundary conditions and comparisons with exact solutions, wherever available. Moreover, several additional variations in the implementation of GA are tried out to tentatively arrive at an optimal variant of the genetic search in terms of the computational speed and accuracy.

\subsection{A method based on energy minimization}

An obvious way to employ a genetic algorithm for response calculations of structural systems is to search for the stationarity (minima) of the associated energy functionals, provided that such functionals can be constructed. The exact asymptotic expression for the total potential energy (Thompson \& Hunt 1984) of an elastic beam (figure 5 below) subjected to axial load may be written as:

$$
\begin{aligned}
V= & \frac{1}{2} E I \int_{0}^{l}\left(w^{\prime \prime 2}+w^{\prime \prime 2} \cdot w^{\prime 2}+w^{\prime \prime 2} \cdot w^{\prime 4}+\ldots\right) \mathrm{d} x \\
& -P \int_{0}^{l}\left(\frac{1}{2} w^{\prime 2}+\frac{1}{8} w^{\prime 4}+\frac{1}{16} w^{\prime 6}+\ldots\right) \mathrm{d} x
\end{aligned}
$$

where, $V$ denotes the total potential energy of the beam, $P$ is the axial compressive load, $w$ is the vertical displacement, $l$ is the length of the beam, $E I$ is the flexural rigidity and

$$
()^{\prime} \equiv \frac{\mathrm{d}}{\mathrm{d} x}() .
$$

For a simply supported beam subjected to axial load $P$, the deflection function can be expanded in terms of the following Ritz basis functions:

$$
w(x)=\sum_{j=1}^{n} A_{j} \sin (j \pi x / l),
$$

where the constants $A_{j}$ constitute the generalized coordinates associated with the $n$ dimensional Hilbert space spanned by $\sin (j \pi x / l), j=1,3,5 \ldots, 2 n-1$. Assuming $j$ to be an odd integer implies that the displacement function is symmetric about the beam centre. Presently, the boundary conditions are:

$$
w(x)=w^{\prime \prime}(x)=0 \quad \text { at } \quad x=0 \quad \text { and } \quad x=l .
$$

Using the approximate function expansion (2), these boundary conditions are automatically satisfied and hence no explicit constraint equation needs to be written out in this case. The 
objective function, $V\left(A_{j} \mid j=1, \ldots n\right)$ may be obtained by substituting (2) in (1). It is however obvious that given the asymptotic and non-quadratic nature of $V$ in (1), the expression for $V$ may become so complicated as to warrant the usage of a symbolic manipulator. In the simplest case of $n=1$, for instance, the expression for $V$ reads as:

$$
\begin{aligned}
V= & \pi^{2}\left[0 \cdot 5 \pi^{2}\left(\frac{1}{2} A_{j}^{2}+\frac{1}{8} \pi^{2} A_{j}^{4}+\frac{1}{16} \pi^{4} A_{j}^{6}+\ldots\right)\right. \\
& \left.-P\left(\frac{1}{4} A_{j}^{2}+\frac{3}{64} \pi^{2} A_{j}^{4}+\frac{5}{256} \pi^{4} A_{j}^{6}+\ldots\right)\right] .
\end{aligned}
$$

At this stage, a global minima of the cost function, $V$, may be approximately determined using a three-operator genetic algorithm (Goldberg 1989) involving reproduction, cross-over and mutation. The search is conducted over a solution space $\left\{A_{j} \mid j=1,3, \ldots, 2 p-1\right\}_{k} \in 3^{p}$, $k=1,2, \ldots, n_{p}$ where $n_{p}$ is the population size used in the global search process.

The second example is concerned with the determination of post-buckled equilibrium paths of a cantilever beam subjected to axial loads. In this case, the deflection function can be expressed asymptotically in terms of the normal mode shapes of a linear cantilever beam. The resulting series is:

$$
w(x)=\sum_{n=1}^{N}\left(C_{1 n} \cosh \left(\beta_{n} x\right)+C_{2 n} \sinh \left(\beta_{n} x\right)+C_{3 n} \cos \left(\beta_{n} x\right)+C_{4 n} \sin \left(\beta_{n} x\right)\right)
$$

where $\beta_{n}=[(2 n-1) / 2 l] \pi$ and $C_{1 n}, C_{2 n}, C_{3 n}$ and $C_{4 n}$ are generalized coordinates to be determined by minimizing the potential energy. The boundary conditions are:

$$
\begin{gathered}
w(x)=w^{\prime}(x)=0, \quad \text { at } \quad x=0, \\
w^{\prime \prime}(x)=w^{\prime \prime \prime}(x)=0, \quad \text { at } \quad x=l .
\end{gathered}
$$

Substituting (5) into the boundary conditions (6) leads to the following constraint identities:

$$
\begin{aligned}
& \sum_{n=1}^{N}\left(C_{1 n}+C_{3 n}\right)=0, \\
& \sum_{n=1}^{N} \beta_{n}\left(C_{2 n}+C_{4 n}\right)=0, \\
& \sum_{n=1}^{N}\left[C_{1 n} \beta_{n}^{2} \cdot \cosh \left(\beta_{n} \cdot l\right)+C_{2 n} \cdot \beta_{n}^{2} \cdot \sinh \left(\beta_{n} l\right)-C_{3 n} \cdot \beta_{n}^{2} \cdot \cos \left(\beta_{n} l\right)\right. \\
& \left.\quad-C_{4 n} \cdot \beta_{n}^{2} \cdot \sin \left(\beta_{n} l\right)\right]=0, \\
& \sum_{n=1}^{N}\left[C_{1 n} \cdot \beta_{n}^{3} \cdot \sinh \left(\beta_{n} \cdot l\right)+C_{2 n} \cdot \beta_{n}^{3} \cdot \cosh \left(\beta_{n} \cdot l\right)+C_{3 n} \cdot \beta_{n}^{3} \cdot \sin \left(\beta_{n} \cdot l\right)\right. \\
& \left.\quad-C_{4 n} \cdot \beta_{n}^{3} \cdot \cos \left(\beta_{n} \cdot l\right)\right]=0 .
\end{aligned}
$$


On substituting the series solution (5) into the general expression for $V$ (as in (1)), one readily obtains the desired objective function $V$ in terms of undetermined generalized coordinates $C_{i n}, i=1, \ldots 4 ; n=1, \ldots, N$.

A genetic search may also be utilized to study the effects of imperfection on the buckling of beams. Thus, allowing for large deflections, the total potential energy of the imperfect beam may be expressed as:

$$
\begin{aligned}
V= & \frac{1}{2} E I \int_{0}^{l}\left[\frac{w_{0}^{\prime \prime}+w^{\prime \prime}}{\left[1-\left(w_{0}^{\prime}+w^{\prime}\right)^{2}\right]^{1 / 2}}-w_{0}^{\prime \prime}\right]^{2} \mathrm{~d} x \\
& -P \int_{0}^{l}\left[1-\left(1-\left[w_{0}^{\prime}+w^{\prime}\right]^{2}\right)^{\frac{1}{2}}\right] \mathrm{d} x,
\end{aligned}
$$

where, $w_{0}(x)$ is the initial imperfection for the beam and $w(x)$ is the additional deflection as measured from the deformed (initially) beam centreline. It is common to study the effects of geometric imperfections by assuming it to be of the following sinusoidal form:

$$
\begin{aligned}
& w_{0}(x)=\delta \sin (\pi x / l) \quad \text { (for a simply supported beam), } \\
& w_{0}(x)=\delta(1-\cos (\pi x / 2 l)) \quad \text { (for a cantilever beam), }
\end{aligned}
$$

where $\delta$ denotes the imperfection amplitude.

In the case of a simply supported beam, the objective function is a function of amplitudes $A_{j}$ and for a clamped-free beam, it is a function of amplitudes $C_{1 n}, C_{2 n}, C_{3 n}$ and $C_{4 n}$. These amplitudes are determined with the help of a genetic algorithm so as to minimize the total potential energy $(V)$. In the present approach, rather than exactly satisfying the equality constraints (7), they are approximated as inequality constraints. In other words, equality constraints are satisfied with an error of the order of $10^{-5}$, say. The inequality constraints imposed on the amplitudes are handled through the "accept or reject" approach. Finally, maximum deflections, for different $P / P_{c r}$ (where $P_{c r}$ is the critical Euler buckling load) values are calculated after substituting the values of $A_{j}$ and $C_{1 n}, C_{2 n}, C_{3 n}$, and $C_{4 n}$ in (2) or (5) as appropriate.

\subsection{A constrained initial value approach}

It is assumed that the equilibrium path of a beam element under prescribed loading and boundary conditions is unique (at least within a local neighbourhood, so that the possibility of multiple solutions is not excluded). The present objective is to appropriately integrate the governing nonlinear ordinary differential equations (ODEs) such that the solution, so found, satisfies all the boundary conditions. Since the governing nonlinear ODEs are generally nonintegrable (so that closed-formed solutions are not available), the most efficient and accurate way to integrate the nonlinear vector fields is to employ some direct integration scheme, such Runge-Kutta, Adams-Bashforth or Newmark schemes. However, in order to effectively employ one of these techniques, sufficient information on initial conditions (at one of the two boundaries) is needed a priori, i.e., BVP needs to be posed as a conditional IVP. To begin with, some of the initial conditions of this conditional IVP are not known and they would have to be optimally selected via a genetic search so as to satisfy all the known boundary conditions. 
For further illustration, consider the following second-order ODEs governing large static deflections of a uniform beam (figure 5a) under in-plane or transversal loading:

$$
\begin{aligned}
& \frac{\mathrm{d}^{2} \theta}{\mathrm{d} s^{2}}+\frac{P}{E I} \sin \theta=0, \quad \text { (for in-plane load) } \\
& \frac{\mathrm{d}^{2} \theta}{\mathrm{d} s^{2}}+\frac{P}{E I} \cos \theta=0, \quad \text { (for transversal load) }
\end{aligned}
$$

where, $\theta, s, P$ and $E I$ are respectively, slope, independent coordinate along the deformed longitudinal axis, axial/lateral load and flexural rigidity of the beam. In order to treat these ODEs as IVPs with $s=0$ (say) as the initial point on the independent axis to start integration, one needs to know the initial conditions $\theta(0) \triangleq \theta_{0}$ and $\theta^{\prime}(0) \triangleq \theta_{0}^{\prime}$ a priori. However, since the ODEs presently are posed as BVPs, only one of these conditions, say $\theta_{0}$, is known at the left boundary $(s=0)$, while other condition, say $\theta^{\prime}(s=l)$, is specified at the right boundary $(s=l)$. Thus the constrained IVP is presently framed by treating $\theta_{0}{ }^{\prime}$ as an unknown variable to be so determined (optimally) that the integrated equilibrium path, $\left\{\theta_{o p t}\left(s, \theta_{0}{ }^{\prime}\right), \theta_{o p t}^{\prime}\left(s, \theta_{0}{ }^{\prime}\right)\right\}$, approaches the known condition $\theta^{\prime}(s=l)$ with a specified error norm. From this viewpoint, the problem again reduces to one of constrained optimization and shall consequently admit a solution based on a genetic search. Let the constrained IVP be approximately integrated via an explicit numerical scheme leading to the local (one-step) maps:

$$
\begin{aligned}
\bar{\theta}_{i+1} & =\Psi_{1}\left(s_{i}, \theta_{i} \triangleq \theta\left(s_{i}\right), \theta_{i}{ }^{\prime} \triangleq \theta^{\prime}\left(s_{i}\right)\right), \\
\bar{\theta}_{i+1}{ }^{\prime} & =\Psi_{2}\left(s_{i}, \theta_{i}, \theta_{i}{ }^{\prime}\right),
\end{aligned}
$$

where $\left(\bar{\theta}_{i+1}, \bar{\theta}_{i+1}^{\prime}\right)$ denote the numerical approximation to $\left(\theta_{i+1}, \theta_{i+1}^{\prime}\right)$, as obtained using the explicit integration scheme, at $s=s_{i+1}$. If the subset $[0, l]$ of the independent axis is discretized into $n$ (say, equal) intervals, then one has via a repeated composition of the above maps:

$$
\begin{aligned}
& \bar{\theta}_{n}=\Psi_{1}^{(n)}\left(0, \theta_{0}, \theta_{0}{ }^{\prime}\right), \\
& \bar{\theta}_{n}{ }^{\prime}=\Psi_{2}^{(n)}\left(0, \theta_{0}, \theta_{0}{ }^{\prime}\right),
\end{aligned}
$$

where, $\Psi_{j}^{(n)}=\Psi_{j^{0}} \Psi_{j^{0}} \ldots \Psi_{j}(n$ times, $j=1,2)$.

Given the known value of $\theta_{n}{ }^{\prime}$, one can therefore construct the following measure for the error to be minimized:

$$
e=\left(\bar{\theta}_{n}{ }^{\prime}-\theta_{n}{ }^{\prime}\right)^{2}=\left\{\Psi_{2}^{(n)}\left(0, \theta_{0}, \theta_{0}{ }^{\prime}\right)-\theta_{n}{ }^{\prime}\right\}^{2} .
$$

For utilizing a genetic algorithm for the above problem, an attempt is made to search for an optimal value, $\theta_{0}{ }^{\prime}(k)$ out of a randomly generated population $\left\{\theta_{0}{ }^{\prime}(j), j=1,2, \ldots n_{p}\right\}$, exploiting the concepts of cross-over and mutation among the members of the population, so that the error, $e$, in (14) reduces to close to zero. Presently a fourth-order explicit RungeKutta method is used for purposes of direct integration. Finally, we get the value of $\theta$ at all the chosen points along the $s$-axis. The Cartesian co-ordinates of the deflected curve at any point $i+1$ is obtained from the relation:

$$
x_{i+1}=x_{i}+\int_{s_{i}}^{s_{i+1}} \cos \theta \mathrm{d} s,
$$




$$
y_{i+1}=y_{i}+\int_{s_{i}}^{s_{i+1}} \sin \theta \mathrm{d} s .
$$

The above integration is accomplished by Simpson's rule.

In addition to the three-operator approach, some other variants of GA such as two-point cross-over, elitist model and variable chromosome lengths are also explored in the search.

\section{Numerical results}

In the following two sub-sections, a few example problems are considered and solved respectively via the energy minimization and the constrained initial value approaches.

\subsection{Energy minimization approach}

For the simply supported case, good convergence is obtained with just three terms in the series, (2). In the energy minimization approach, each undetermined generalized co-ordinate is represented by 8 bits. The results obtained via the present approach are compared with those via the classical approach in table 1 for both simply supported and cantilever beams. It may be observed from the table that the results compare well. The same has been shown graphically in figures 1 and 2 . However it may be noted that the deflections obtained from the present method differ from reference values by as much as $2.2 \%$ in the case of simply supported beam and $2.6 \%$ in the case of clamped-free beam for $P / P_{c r}=4.02$. The load-deflection path for an imperfect beam, with different value of $\delta$, is shown in figure 3 for the simply supported case and in figure 4 for the fixed-free case. Schematic representation of deflected shape of a simply supported beam is shown in figure 5a. As the imperfection amplitude increases, the beam deflects more for the same intensity of load. Deflection curves of a simply supported beam for various value of $P / P_{c r}$ are shown in figure $5 \mathrm{~b}$. In all the above computation, the

Table 1. Comparisons of present deflected values with those based on a classical approach for different values of axial load.

\begin{tabular}{|c|c|c|c|c|}
\hline \multirow[b]{2}{*}{$P / P_{c r}$} & \multicolumn{2}{|c|}{$\begin{array}{l}\text { Simply supported beam } \\
\text { (central deflection) }\end{array}$} & \multicolumn{2}{|c|}{$\begin{array}{l}\text { Cantilever beam } \\
\text { (tip deflection) }\end{array}$} \\
\hline & $\begin{array}{l}\text { Present } \\
\text { approach }\end{array}$ & $\begin{array}{c}\text { Classical } \\
\text { approach } \\
\text { (Timenshenko 1963) }\end{array}$ & $\begin{array}{l}\text { Present } \\
\text { approach }\end{array}$ & $\begin{array}{c}\text { Classical } \\
\text { approach } \\
\text { (Timenshenko 1963) }\end{array}$ \\
\hline 1.00 & 0.000 & 0.000 & 0.000 & 0.000 \\
\hline 1.015 & $0 \cdot 109$ & $0 \cdot 110$ & $0 \cdot 217$ & 0.220 \\
\hline 1.063 & 0.210 & 0.211 & 0.418 & 0.422 \\
\hline $1 \cdot 152$ & 0.295 & 0.297 & 0.590 & 0.593 \\
\hline 1.293 & $0 \cdot 357$ & $0 \cdot 360$ & 0.708 & 0.719 \\
\hline 1.518 & 0.392 & 0.396 & 0.786 & 0.792 \\
\hline 1.884 & 0.397 & 0.402 & 0.799 & 0.803 \\
\hline $2 \cdot 541$ & $0 \cdot 366$ & $0 \cdot 375$ & 0.731 & 0.750 \\
\hline 4.029 & 0.306 & 0.313 & 0.609 & 0.625 \\
\hline
\end{tabular}




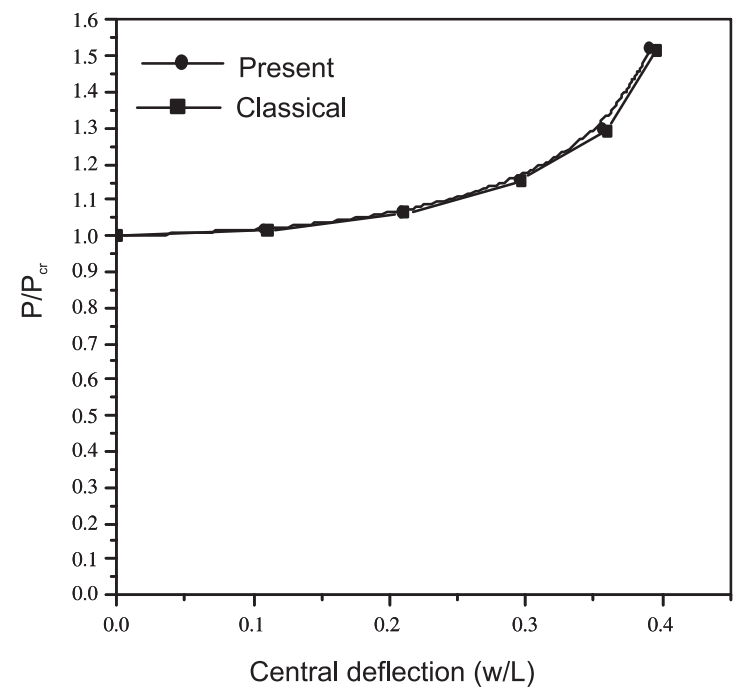

Figure 1. Equilibrium path of a simply supported beam.

population size has been kept at 50 and $P_{\text {cross }}=0.80 ; P_{\text {mut }}=0.002$; for the three-operator genetic algorithm.

The frequency with which the genetic material is exchanged and the initial population size have significant effects on the results. There are certain optimal values for each problem. In the present case, the cross over and mutation probability along with the initial population size are varied to approximately obtain the optimal values of $p_{\text {cross }}$ and $p_{\text {mut }}$. Table 2 illustrates the influence of $p_{\text {cross }}$ and $p_{\text {mut }}$ on the minimization of the potential energy. Additionally, effect of one- and two-point cross over as well as the incorporation of an elitist model (Goldberg

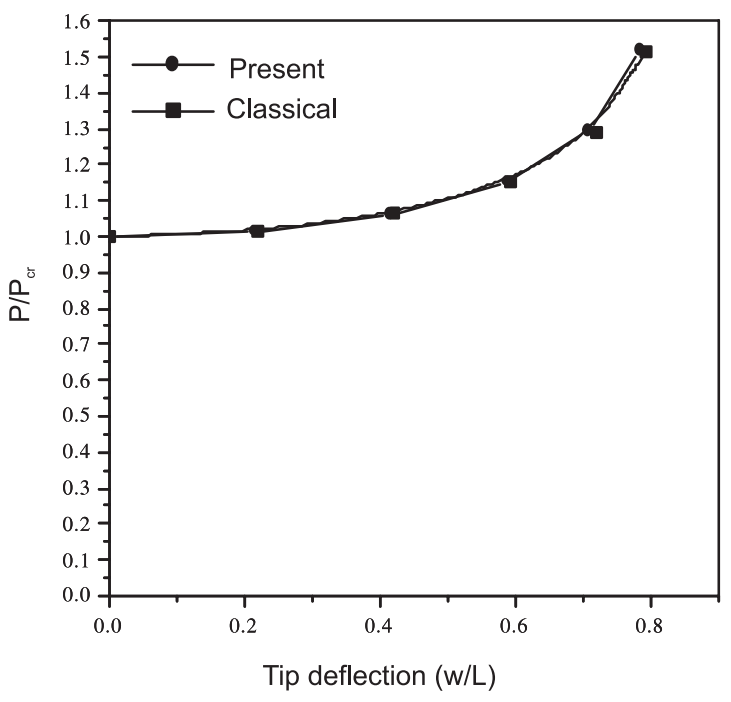

Figure 2. Equilibrium path of a cantilever beam. 


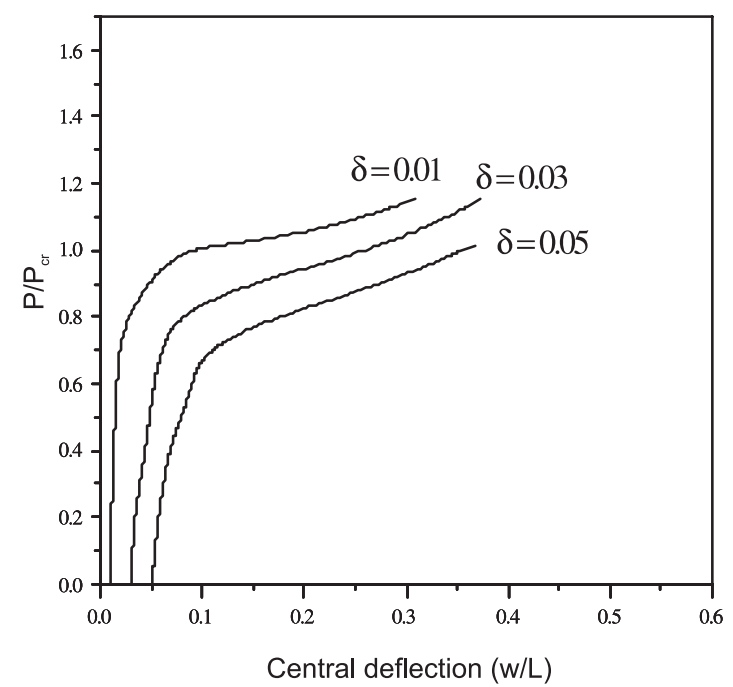

Figure 3. Equilibrium path of an imperfect simply supported beam for different amplitudes of imperfection.

1999) are also documented in this table. The idea behind the elitist operator is to keep the best chromosome found in the initial population in the population pool and to protect it from destruction due to cross over and mutation. This chromosome is replaced only when a better chromosome is found in the subsequent generation. The same starting seed is used in all calculations and the maximum number of generations is arbitrarily restricted to 100. GA contains a multitude of parameters, which need to be tuned to improve the performance, i.e. faster convergence to an optimal solution. In addition to the parameters already discussed, the influence of seed, number of generations and the length of chromosomes on the optimum are studied. Some of them are reported in tables 3 and 4. Similarly for a cantilever, three

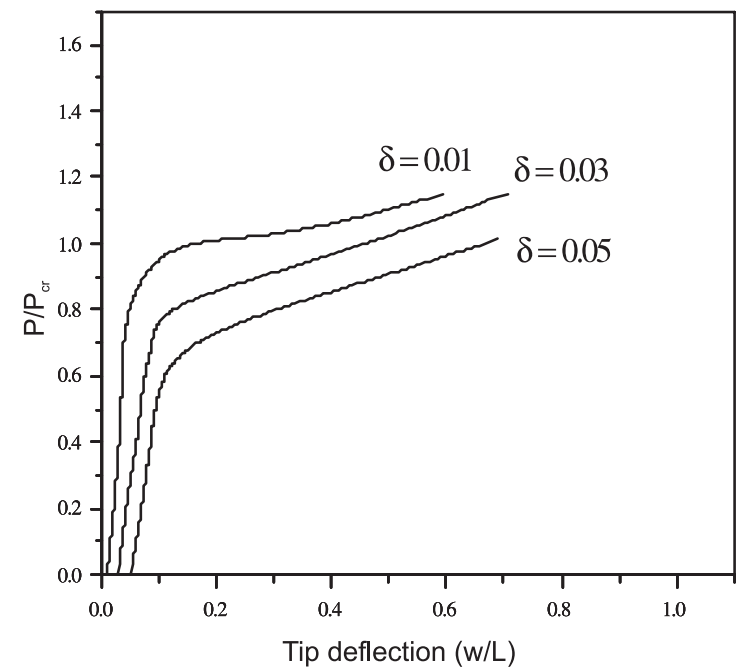

Figure 4. Equilibrium path of an imperfect cantilever beam for different amplitudes of imperfection. 


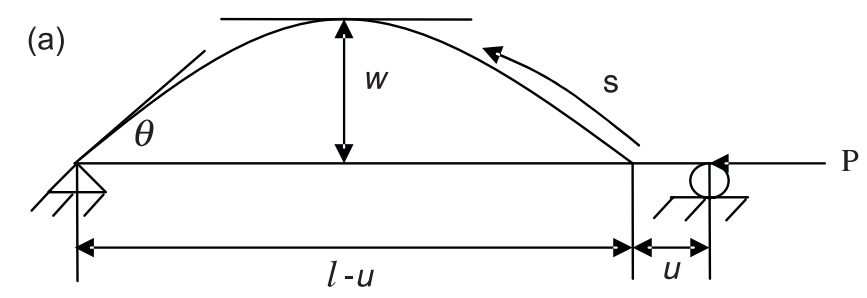

(b)

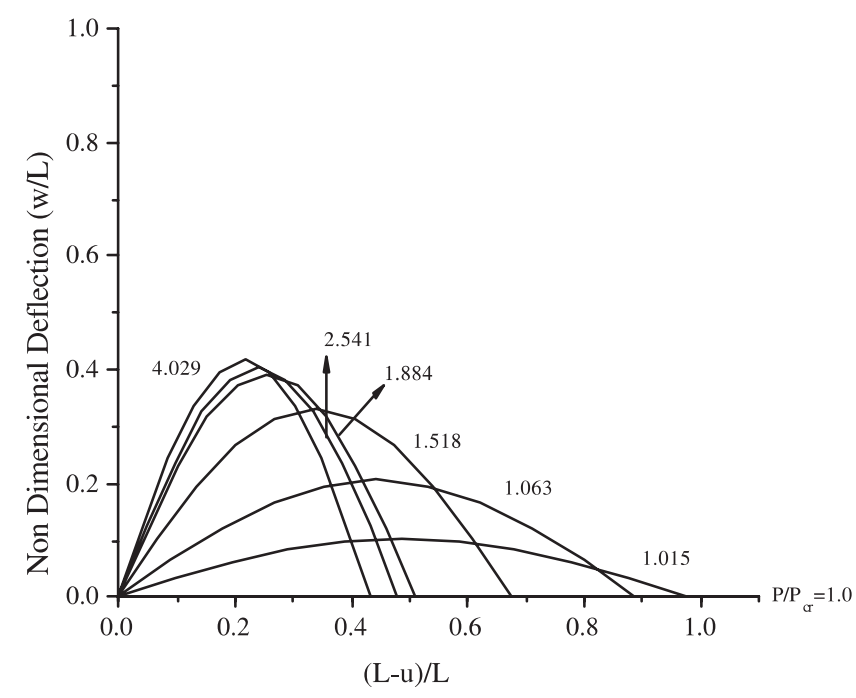

Figure 5. (a) Schematic representation of deformed shape of simply supported beam; $u, w, l$ and $p$ are respectively, axial displacement, lateral deflection, length and axial load for the beam. (b) Deflected shape of the simply supported beam at different levels of axial loads.

terms in the series (5) yield a good convergence. After 65 generations there is apparently no improvement in the objective function.

\subsection{Constrained initial value approach}

The constrained initial value approach, to the knowledge of the authors, is being proposed for the first time and is presently used to solve several beam problems. These include a simply supported beam subjected to an axial load, a clamped-free beam subjected to an axial load and finally a clamped-free beam subjected to a transverse load at the tip. Results obtained by the present approach are compared with those via a classical approach in table 5 for both simply supported and cantilever beams subjected to axial loads. The results are observed to be in good agreement. The maximum absolute difference in results between present approach and classical approach is $1.29 \%$ for simply supported and $2.12 \%$ for clamped-free beam (for $\left.P / P_{c r}=4.02\right)$. Compared with the first approach, errors in the second approach appear to have reduced. Deflection curves of an axially loaded cantilever beam for various values of $P / P_{c r}$ have been shown in figure 6. Lateral deformation $(w)$, axial deformation $(u)$ and slope at the tip $(\alpha)$ of a tip-loaded cantilever beam are shown in table 6 along with the so-called exact values. It may be observed from this table that the results do compare well. The relevant deflected shape plots are shown in figure 7. Deflection curves of tip-loaded cantilever beam for various values of $P L^{2} / E I$ have been shown in figure 8 . 
Table 2. Influence of $P_{\text {cross }}$ and $P_{\text {mut }}$ on the optimization process (seed no. 234567, $P / P_{c r}=1.015$, pop. size $=50$ and max. gen. $=100$ ).

$\mathrm{R}$ - reproduction, $\mathrm{C}$ - cross-over, $\mathrm{M}$ - mutation, TPC - two point cross-over, $\mathrm{E}$ - elitist model

\begin{tabular}{|c|c|c|c|c|c|c|}
\hline \multirow[b]{2}{*}{$P_{\text {cross }}$} & \multirow[b]{2}{*}{$P_{\text {mut }}$} & \multicolumn{2}{|c|}{ Simply supported beam } & \multicolumn{2}{|c|}{ Cantilever beam } & \multirow[b]{2}{*}{$\begin{array}{c}\text { Active } \\
\text { operators }\end{array}$} \\
\hline & & $\begin{array}{l}\text { Central } \\
\text { deflection } \\
(w / L)\end{array}$ & $\begin{array}{c}\text { Generation } \\
\text { No. }\end{array}$ & $\begin{array}{l}\text { Tip } \\
\text { deflection } \\
(w / L)\end{array}$ & $\begin{array}{c}\text { Generation } \\
\text { No. }\end{array}$ & \\
\hline 0.7 & 0.002 & $0 \cdot 10877$ & 76 & $0 \cdot 21679$ & 80 & \multirow{3}{*}{$\mathrm{R}+\mathrm{C}+\mathrm{M}$} \\
\hline $0 \cdot 8$ & 0.002 & $0 \cdot 10875$ & 73 & $0 \cdot 21676$ & 77 & \\
\hline 0.9 & $0 \cdot 002$ & $0 \cdot 10877$ & 70 & $0 \cdot 21675$ & 78 & \\
\hline 0.7 & 0.002 & $0 \cdot 10879$ & 68 & $0 \cdot 21677$ & 75 & \multirow{3}{*}{$\mathrm{R}+\mathrm{TPC}+\mathrm{M}$} \\
\hline 0.8 & 0.003 & $0 \cdot 10875$ & 66 & 0.21678 & 77 & \\
\hline 0.9 & $0 \cdot 002$ & $0 \cdot 10879$ & 65 & $0 \cdot 21679$ & 74 & \\
\hline 0.7 & 0.002 & $0 \cdot 10876$ & 62 & $0 \cdot 21675$ & 70 & \multirow{3}{*}{$\mathrm{R}+\mathrm{C}+\mathrm{M}+\mathrm{E}$} \\
\hline $0 \cdot 8$ & 0.003 & 0.10878 & 60 & 0.21676 & 67 & \\
\hline 0.9 & 0.002 & $0 \cdot 10879$ & 58 & $0 \cdot 21676$ & 67 & \\
\hline
\end{tabular}

Table 3. Influence of seed number on the optimization process $\left(P / P_{c r}=1 \cdot 015\right.$, pop. size $=50$ and max. gen. $=100$ ).

\begin{tabular}{|c|c|c|c|c|c|c|}
\hline \multirow[b]{2}{*}{$\begin{array}{l}\text { Seed } \\
\text { No. }\end{array}$} & \multirow[b]{2}{*}{$P_{\text {cross }}$} & \multirow[b]{2}{*}{$P_{\mathrm{mut}}$} & \multicolumn{2}{|c|}{ Simply supported beam } & \multicolumn{2}{|c|}{ Clamped beam } \\
\hline & & & $\begin{array}{l}\text { Central } \\
\text { deflection } \\
(w / L)\end{array}$ & $\begin{array}{c}\text { Generation } \\
\text { No. }\end{array}$ & $\begin{array}{l}\text { Tip } \\
\text { deflection } \\
(w / L)\end{array}$ & $\begin{array}{c}\text { Generation } \\
\text { No. }\end{array}$ \\
\hline 234567 & $0 \cdot 80$ & 0.002 & $0 \cdot 10875$ & 73 & $0 \cdot 21676$ & 77 \\
\hline 123456 & $0 \cdot 80$ & 0.002 & $0 \cdot 10878$ & 75 & 0.21679 & 78 \\
\hline 345678 & $0 \cdot 80$ & 0.002 & $0 \cdot 10876$ & 65 & 0.21675 & 70 \\
\hline 542345 & 0.80 & 0.002 & $0 \cdot 10875$ & 68 & $0 \cdot 21677$ & 72 \\
\hline
\end{tabular}

Table 4. Influence of population size on the optimization process $\left(P / P_{c r}=1 \cdot 015\right.$, max. gen. $=100$, seed no. 234567).

\begin{tabular}{|c|c|c|c|c|c|c|}
\hline \multirow[b]{2}{*}{$\begin{array}{l}\text { Population } \\
\text { size }\end{array}$} & \multirow[b]{2}{*}{$P_{\text {cross }}$} & \multirow[b]{2}{*}{$P_{\text {mut }}$} & \multicolumn{2}{|c|}{ Simply supported beam } & \multicolumn{2}{|c|}{ Clamped beam } \\
\hline & & & $\begin{array}{l}\text { Central } \\
\text { deflection } \\
(w / L)\end{array}$ & $\begin{array}{c}\text { Generation } \\
\text { No. }\end{array}$ & $\begin{array}{l}\text { Tip } \\
\text { deflection } \\
(w / L)\end{array}$ & $\begin{array}{c}\text { Generation } \\
\text { No. }\end{array}$ \\
\hline 50 & 0.80 & 0.002 & 0.10875 & 73 & 0.21676 & 77 \\
\hline 100 & 0.80 & 0.002 & 0.10872 & 61 & 0.21678 & 63 \\
\hline 150 & 0.80 & 0.002 & 0.10869 & 45 & 0.21688 & 45 \\
\hline 200 & $0 \cdot 80$ & 0.002 & $0 \cdot 10870$ & 33 & $0 \cdot 21671$ & 39 \\
\hline
\end{tabular}


Table 5. Comparison of present results with the classical approach results for simply supported and cantilever beams by constrained initial value approach.

\begin{tabular}{lccccc}
\hline & \multicolumn{2}{c}{ Central deflection $(w / L)$} & & \multicolumn{2}{c}{ Tip deflection $(w / L)$} \\
\cline { 2 - 3 } \cline { 5 - 5 }$P / P_{c r}$ & $\begin{array}{c}\text { Present } \\
\text { approach }\end{array}$ & $\begin{array}{c}\text { Classical } \\
\text { approach } \\
\text { (Timenshenko 1963) }\end{array}$ & & $\begin{array}{c}\text { Present } \\
\text { approach }\end{array}$ & $\begin{array}{c}\text { Classical } \\
\text { approach } \\
\text { (Timenshenko 1963) }\end{array}$ \\
\hline 1.00 & $0 \cdot 000$ & 0.000 & & 0.000 & 0.000 \\
1.015 & $0 \cdot 109$ & 0.110 & & 0.219 & 0.220 \\
1.063 & 0.209 & 0.211 & & 0.421 & 0.422 \\
1.152 & 0.296 & 0.297 & & 0.592 & 0.593 \\
1.293 & 0.358 & 0.360 & & 0.715 & 0.719 \\
1.518 & 0.393 & 0.396 & & 0.787 & 0.792 \\
1.884 & 0.399 & 0.402 & & 0.796 & 0.803 \\
2.541 & 0.370 & 0.375 & & 0.737 & 0.750 \\
4.029 & 0.309 & 0.313 & 0.612 & 0.625 \\
\hline
\end{tabular}

The constrained initial value approach turns out to be a considerably faster algorithm than its energy minimization counterpart. In fact, the relative speed of the initial value algorithm sharply increases with the increase in the population size and/or dimensionality of the optimization problem. The CPU times taken by the first and second approaches for a typical problem (for cantilever beam subjected to an axial load) are shown in table 7. Moreover, the CPU time differences between the energy minimization and the initial value algorithms are plotted on a function of the population size in figure 9. The near-exponential growth in CPU

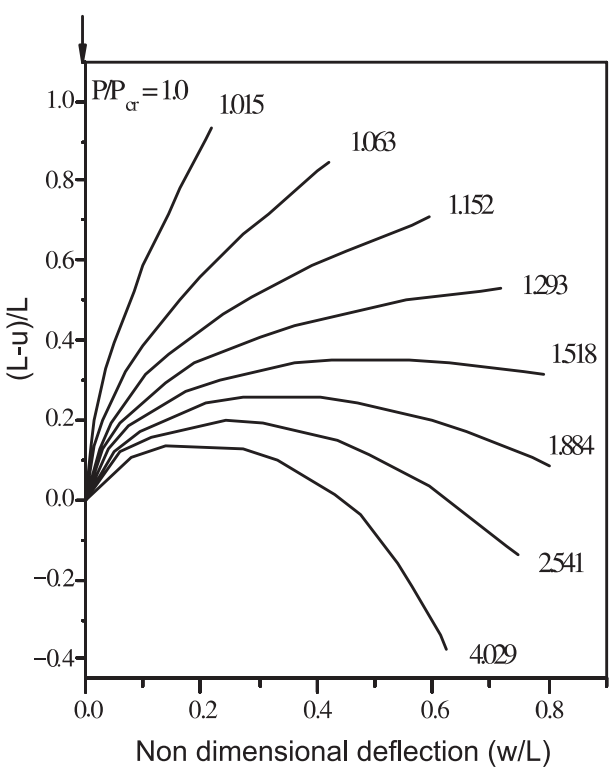

Figure 6. Deflected shape of cantilever column under levels of axial load. 


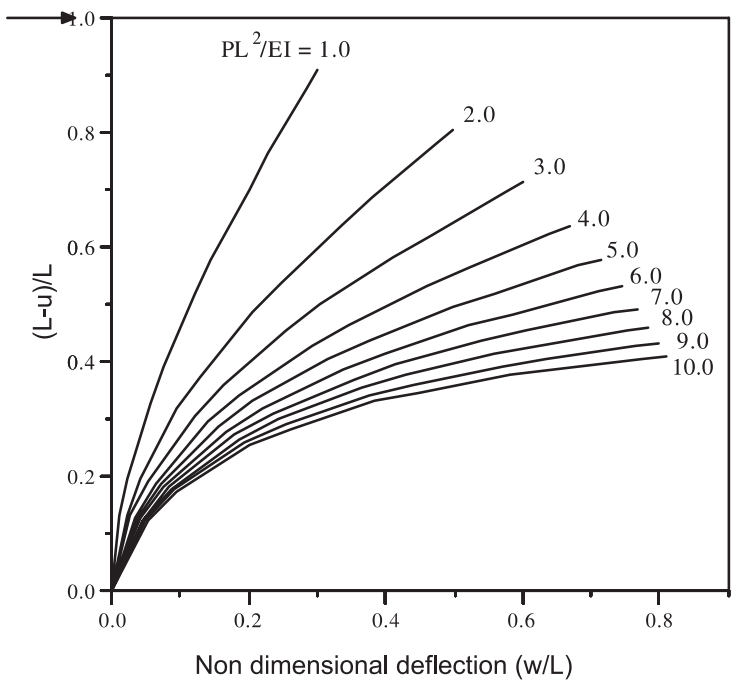

Figure 7. Deflected shape of cantilever beam (subjected to tip load) at different values of $P / P_{c r}$.

time via the energy minimization algorithm relative to that via the initial value algorithm is an indicator of the superior computational efficiency of the latter. As a final numerical experiment, the initial value approach is implemented with a variable chromosome length, i.e, 8 bits for the first 50 generations and 12 bits thereafter. While choosing a consistent length of 8 bit throughout is a computationally faster and less accurate option, a 12-bit chromosome length, on the other hand, is obviously a slower and more accurate alternative. The variable length approach turns strives to strike a balance between the two. As shown in table 8, the variable length approach offers a much better alternative w.r.t. its constant length counterpart, both in terms of speed and accuracy.

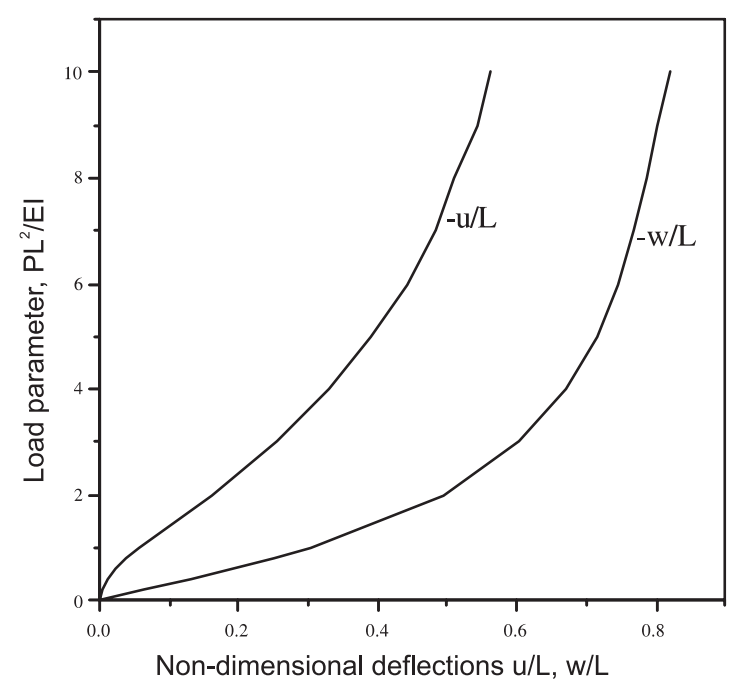

Figure 8. Load-deflection curves for the cantilever beam subjected to transversal load at tip. 
Table 6. Deflections and slopes of a tip loaded cantilever beam.

\begin{tabular}{cccccccc}
\hline & \multicolumn{3}{c}{ Present approach } & & \multicolumn{3}{c}{ Exact (Mattiason 1981) } \\
\cline { 2 - 3 } \cline { 6 - 7 }$P L^{2} / E I$ & $w / L$ & $u / L$ & $\alpha$ & & $w / L$ & $u / L$ & $\alpha$ \\
\hline 1.0 & 0.30192 & 0.05662 & 0.47212 & & 0.30172 & 0.05643 & 0.46135 \\
$2 \cdot 0$ & 0.49392 & 0.16160 & 0.79274 & & 0.49346 & 0.16064 & 0.78175 \\
$3 \cdot 0$ & 0.60389 & 0.25541 & 0.99005 & & 0.60325 & 0.25442 & 0.98602 \\
$4 \cdot 0$ & 0.67042 & 0.32925 & 1.14256 & & 0.66996 & 0.32894 & 1.12124 \\
$5 \cdot 0$ & 0.71462 & 0.38825 & 1.23434 & & 0.71379 & 0.38763 & 1.21537 \\
$6 \cdot 0$ & 0.74546 & 0.44239 & 1.29730 & & 0.74457 & 0.43459 & 1.28370 \\
$7 \cdot 0$ & 0.76821 & 0.48211 & 1.34236 & & 0.76737 & 0.47293 & 1.33496 \\
$8 \cdot 0$ & 0.78612 & 0.5110 & 1.38545 & & 0.78498 & 0.50483 & 1.37443 \\
$9 \cdot 0$ & 0.80305 & 0.54281 & 1.41568 & & 0.79906 & 0.53182 & 1.40547 \\
$10 \cdot 0$ & 0.82065 & 0.56215 & 1.44687 & & 0.81061 & 0.55500 & 1.43029 \\
\hline
\end{tabular}

Table 7. CPU times required by the two approaches (for calculating the tip deflection of a cantilever beam subjected to an axial load).

\begin{tabular}{lccccc}
\hline & \multicolumn{2}{c}{ First approach } & & \multicolumn{2}{c}{ Second approach } \\
\cline { 2 - 3 } \cline { 5 - 6 }$P L^{2} / E I$ & $\begin{array}{c}\text { Tip deflection } \\
(w / L)\end{array}$ & $\begin{array}{c}\text { CPU time } \\
\text { (in min) }\end{array}$ & & $\begin{array}{c}\text { Tip deflection } \\
(w / L)\end{array}$ & $\begin{array}{c}\text { CPU time } \\
\text { (in min) }\end{array}$ \\
\hline 1.015 & 0.217 & 1.12 & & 0.219 & 0.22 \\
1.063 & 0.418 & 1.12 & & 0.421 & 0.22 \\
1.152 & 0.590 & 1.12 & & 0.592 & 0.23 \\
1.293 & 0.708 & 1.13 & & 0.715 & 0.22 \\
1.518 & 0.786 & 1.13 & & 0.787 & 0.24 \\
1.884 & 0.799 & 1.13 & & 0.796 & 0.23 \\
2.541 & 0.731 & 1.14 & & 0.737 & 0.23 \\
4.029 & 0.609 & 1.14 & & 0.612 & 0.24
\end{tabular}

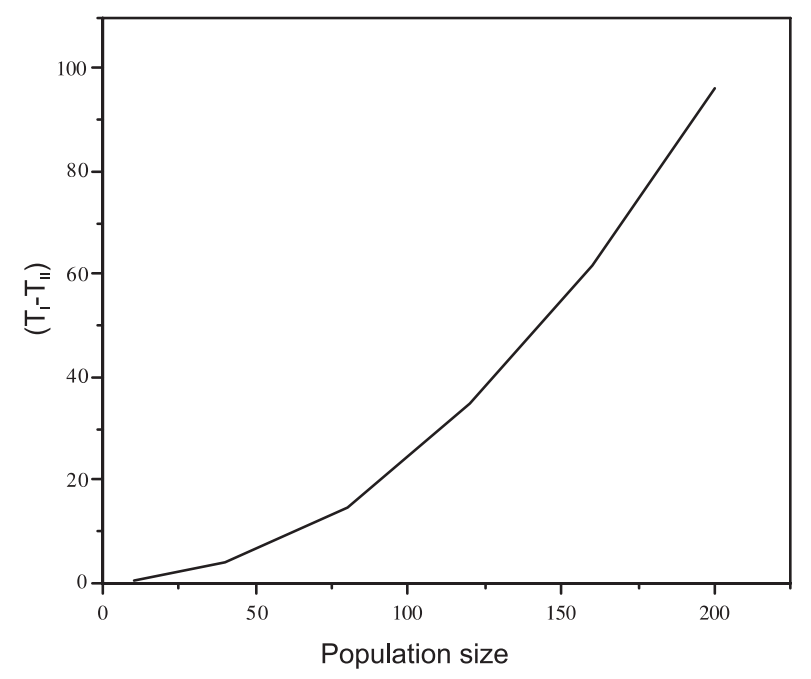

Figure 9. CPU time difference (between two approaches) vs population size; cantilever beam. $T_{I}=$ time required for an energy minimization approach; $T_{I I}=$ time required for the constrained initial value approach. 
Table 8. Influence of chromosome length on the optimization process $\left(P / P_{c r}=1 \cdot 015\right.$, $\max$. gen. $=100$, seed $123456, P_{\text {cross }}=0 \cdot 8, P_{\text {mut }}=0 \cdot 002$, pop. size $\left.=50\right)$.

\begin{tabular}{|c|c|c|c|c|c|c|}
\hline $\begin{array}{l}\text { Chromosome } \\
\text { length }\end{array}$ & $\begin{array}{l}\text { Central } \\
\text { deflection } \\
(w / L)\end{array}$ & $\begin{array}{l}\text { Gen.* } \\
\text { No. }\end{array}$ & $\begin{array}{c}\text { Exact } \\
\text { (Timoshenko } \\
\text { 1963) }\end{array}$ & $\begin{array}{c}\text { Tip } \\
\text { deflection } \\
(w / L)\end{array}$ & $\begin{array}{l}\text { Gen.* } \\
\text { No. }\end{array}$ & $\begin{array}{c}\text { Exact } \\
\text { (Timoshenko } \\
\text { 1963) }\end{array}$ \\
\hline $\begin{array}{l}\text { Constant } \\
\text { chromosome } \\
\text { of length } 8\end{array}$ & $0 \cdot 10910$ & 89 & $0 \cdot 110$ & $0 \cdot 21745$ & 91 & $0 \cdot 220$ \\
\hline $\begin{array}{l}\text { Constant } \\
\text { chromosome } \\
\text { of length } 12\end{array}$ & $0 \cdot 10941$ & 80 & $0 \cdot 110$ & $0 \cdot 21763$ & 83 & $0 \cdot 220$ \\
\hline $\begin{array}{l}\text { Variable } \\
\text { chromosome } \\
\text { of length } 8 \text { and } 12\end{array}$ & $0 \cdot 10957$ & 78 & $0 \cdot 110$ & $0 \cdot 21782$ & 72 & 0.220 \\
\hline
\end{tabular}

${ }^{*}$ Gen. - generation

\section{Conclusions}

In this study the equilibrium paths of nonlinear beams under axial or transverse loads, with or without geometric imperfections and different boundary conditions, are obtained via a couple of genetic search techniques. While the first approach is a straightforward application of the principle that equilibrium configurations correspond to stationary energy values, the second approach is based on a shooting strategy, which converts a BVP to a constrained IVP. The second approach, in particular, offers quite an attractive computational alternative given the fact that there exists a host of efficient numerical integrators for direct integration of nonlinear ordinary differential equations. Correspondingly, the second approach not only constitutes an accurate scheme, but also a computationally faster strategy to obtain equilibrium solutions within a genetic search framework. An additional advantage of the second approach is that, it does not need an associated energy functional for obtaining the solution, which is of distinct advantage, especially in such cases as beams under follower forces and other non-conservative loading conditions. It is observed from numerical results that, while both the methods yield reasonably accurate results, the second approach offers a more efficient and elegant alternative. It would of considerable interest to apply these procedures in the context of finite-rotation (kinematically exact) beam theories (Simo 1992) and simulations of nonlinear beam dynamics governed by partial differential equations.

\section{References}

Arora J S 1989 Introduction to optimum design (New York: Mcgraw-Hill)

Barten H J 1945 On the deflection of a cantilever beams. Q. Appl. Math. 3: 275-276

Bishop K E, Drucker D C 1945 Large deflection of cantilever beams. Appl. Math. 3: 272-275

Goldberg D E 1989 Genetic algorithms in search, optimization and machine learning (Reading, MA: Addison-Wesley)

Ibrahimbegovic A, Shakourzadeh H, Batoz J L, Guo Y-K 1996 On the role of geometrically exact and second-order theories in buckling and post-buckling analysis of three dimensional beam structures. Comput. Struct. 61: 1101-1114 
Kuznetsov V V, Levyakov S V 2002 Complete solution of the stability problem for elastica of Euler's column. Int. J. Nonlinear Mech. 37: 1003-1009

Lee B K, Wilson J F, Oh S J 1993 Elastica of cantilevered beams with variable cross sections. Int. J. Nonlinear Mech. 28: 579-589

Mattiasson K 1981 Numerical results from large deflection beam and frame problems analyzed by means of elliptic integrals. Int. J. Numer. Methods Engng. 17: 145-153

Ramachandra L S, Roy D 2001a A new method for two-point nonlinear boundary value problems in solid mechanics. J. Appl. Mech. 68: 776-786

Ramachandra L S, Roy D 2001b A novel technique in the solution of axisymmetric large deflection analysis of circular plates. J. Appl. Mech. 68: 814-816

Rao S S 1984 Optimization theory and applications 2nd edn, (New Delhi: Wiley Eastern).

Stoker J J 1992 Nonlinear vibrations in mechanical and electrical systems (Somerset, NJ: John Wiley \& Sons)

Simo J C 1992 The (symmetric) Hessian for geometrically nonlinear models in solid mechanics: intrinsic definition and geometric interpretation. Comput. Methods Appl. Mech. Engng. 96: 189200

Simo J C, Vu-Quoc L 1986 A three-dimensional finite-strain rod model. Part II: computational aspects. Comput. Methods. Appl. Mech. Engng. 58: 79-116

Thompson J M T, Hunt G W 1984 Elastic instability phenomena (Chichester: Wiley)

Timoshenko S P, Gere J M 1963 Theory of elastic stability (New York: McGraw-Hill)

Vaz M A, Silva D F C 2003 Post-buckling analysis of slender elastic rods subjected to terminal forces. Int. J. Nonlinear Mech. 38: 483-492 